\title{
Les peptidases des lactocoques
}

\author{
V Monnet, MP Chapot-Chartier, JC Gripon
}

Unité d'enzymologie, station de recherches laitières, INRA, 78352 Jouy-en-Josas, France

\begin{abstract}
Résumé - Les lactocoques sont exigeants sur le plan nutritionnel et sont auxotrophes pour certains acides aminés. Pour trouver l'azote aminé nécessaire à leur croissance optimale, ces bactéries doivent hydrolyser les protéines du lait. Une protéinase liée aux enveloppes cellulaires (maintenant bien caractérisée) libère des peptides de taille supérieure à 5 résidus. Ces derniers sont hydrolysés par des peptidases en acides aminés libres et en petits peptides capables d'être transportés à travers la membrane par des systèmes de transport actif. Outre leur rôle dans la nutrition azotée, les peptidases permettent, dans les fromages, l'hydrolyse des peptides amers et la libération d'acides aminés libres et de petits peptides, précurseurs de composés d'arômes. Le système peptidasique des lactocoques comprend au moins 3 aminopeptidases, une dipeptidase, une tripeptidase, une $X$ prolyl dipeptidyl aminopeptidase, une prolidase, et 3 endopeptidases. Actuellement, les gènes codant pour 3 peptidases différentes ont été clonés et séquencés. Il s'agit des gènes pep $N$ et pepC codant chacun pour une aminopeptidase de spécificité large, et du gène pepX codant pour la X-prolyl dipeptidyl aminopeptidase. Les propriétés, la localisation cellulaire et le rôle de ces enzymes dans la nutrition azotée des lactocoques sont discutés dans cette revue.
\end{abstract}

protéolyse / peptidases / nutrition azotée / Lactococcus lactis

Summary - Lactococcal peptidases. Lactococci are nutritionally fastidious and are auxotrophic for several amino acids. In order to find the nitrogen source necessary to grow to high cell density, these bacteria need to hydrolyze milk proteins. A cell envelope-associated proteinase (which is now well characterized) releases peptides composed of $>5$ residues. These peptides are further hydrolyzed into free amino acids and small peptides which can be transported through the cytoplasmic membrane by active transport systems. In addition to their role in nitrogen supply, peptidases are responsible for the degradation of bitter peptides in cheese and for the release of free amino acids and small peptides which are precursors of aromatic compounds. The lactococcal peptidasic system is composed of at least 3 aminopeptidases, one dipeptidase, one tripeptidase, one X-prolyl-dipeptidylaminopeptidase, one prolidase and 3 endopeptidases. Up to now, the genes coding for 3 different peptidases have been cloned and sequenced: pepN and pepC, each of them encoding an aminopeptidase with broad specificity, and pepX encoding the X-prolyl-dipeptidyl-aminopeptidase. The properties, the cellular location and the role of these enzymes in bacterial nutrition are discussed in this review.

proteolysis / peptidases / nitrogen nutrition / Lactococcus lactis 


\section{INTRODUCTION}

Les premières études concernant la mise en évidence d'activités peptidasiques chez les bactéries lactiques ainsi que la caractérisation des enzymes impliquées sont déjà assez anciennes (Mou et al, 1975; Desmazeaud and Zevaco, 1977; Schmidt et al, 1977; Law, 1979; Cliffe et Law, 1979; Kaminogawa et al, 1984). Cependant, l'amélioration constante des techniques de séparation des protéines d'une part, et le développement des techniques de biologie moléculaire appliquées aux bactéries lactiques d'autre part, ont récemment donné un nouvel essor à l'étude des peptidases des bactéries lactiques. Les données accumulées sur la protéinase extracellulaire des lactocoques et les peptides qu'elle libère ont également été une incitation à la recherche de peptidases impliquées dans la suite du processus de protéolyse chez les lactocoques (tableau I).

Les lactocoques sont exigeants sur un plan nutritionnel et sont auxotrophes pour au moins 4 acides aminés (His, Ile, Leu, Val) (Chopin, communication personnelle). Ces bactéries doivent donc trouver dans le lait l'azote aminé qui leur est nécessaire et pour cela hydrolyser les protéines du lait. II est maintenent bien établi qu'une protéinase liée aux enveloppes cellulaires est indispensable à la croissance optimale des lactocoques sur lait (Thomas et Mills, 1981). Elle a été bien caractérisée tant sur le plan biochimique que génétique (revue de Thomas et Pritchard, 1987 ; revue de Kok \& Venema, 1988). Elle hydrolyse les caséines et libère des peptides qui sont généralement hydrophobes, riches en proline et trop longs pour pouvoir traverser la membrane plasmique (Monnet et al, 1986, 1989, 1992; Visser et al, 1988, 1991; Reid et al, 1991a et b). La nutrition azotée des lactocoques et par conséquent leur croissance optimale passe donc par une hy- drolyse plus poussée de ces peptides par des peptidases. Outre leur rôle dans la nutrition azotée, les peptidases permettent, dans un contexte fromager, I'hydrolyse des peptides amers et la libération d'acides aminés libres et de petits peptides, précurseurs de composés d'arôme. Elles participent donc aussi à l'apparition recherchée des qualités organoleptiques des fromages. Pour ces 2 raisons, de nombreuses équipes cherchent à isoler, caractériser ces peptidases et à les modifier ou à amplifier leur activité en vue de construire des souches de lactocoques plus performantes.

\section{AMINOPEPTIDASES}

\section{Caratérisation biochimique des enzymes}

Les aminopeptidases sont les peptidases de lactocoques les plus étudiées, sans doute parce que leur détection a été, au départ, facilitée par l'emploi de substrats chromogènes (acides aminés p-nitroanilide ou $\beta$-naphtylamide). Trois aminopeptidases ont plus spécialement été isolées et caractérisées chez les lactocoques. Deux d'entre elles ont une spécificité large mais sont cependant clairement différentes. L'aminopeptidase $\mathrm{N}(\mathrm{PepN})$, isolée de la souche de Lactococcus lactis subsp cremoris Wg2, est une métalloenzyme monomérique d'un poids moléculaire de $95 \mathrm{kDa}$ (Tan et Konings, 1990). Elle semble similaire à celles isolées par Desmazeaud et Zevaco (1979) à partir d'une souche de $L$ lactis subsp diacetylactis et par Geis et al (1985) à partir d'une souche de L lactis subsp cremoris AC1. L'aminopeptidase C $(\mathrm{PepC})$ est une enzyme à cystéine, isolée de la souche $L$ lactis subsp cremoris AM2, ayant une structure hexamérique et un poids moléculaire de $300 \mathrm{kDa}$ (Neviani et 
al, 1989). L'aminopeptidase A, quant à elle, présente une spécificité étroite puisqu'elle ne libère que les résidus Asp et Glu en position $\mathrm{N}$-terminale. C'est une métalloenzyme isolée de $L$ lactis subsp lactis NCDO 712 par Niven (1991) qui lui attribue un poids moléculaire de $245 \mathrm{kDa}$ et une structure hexamérique et par Exterkate et de Veer (1987a) à partir de la souche $L$ lactis subsp cremoris HP; ces derniers la décrivent comme un trimère de $130 \mathrm{kDa}$ associé à la membrane. Les 2 enzymes décrites sont très thermostables et seules des études complémentaires comme l'établissement de la séquence de leur gène permettraient de savoir s'il s'agit de la même protéine.

\section{Clonage et séquençage des gènes}

Actuellement, les gènes codant pour 3 peptidases différentes ont été clonés et séquencés. Deux d'entre eux concernent des aminopeptidases. II s'agit des gènes pepN et pepC, codant pour chacune des 2 aminopeptidases de spécificité large.

Le gène $p e p N$ a été cloné et séquencé dans 2 souches différentes de $L$ lactis subsp cremoris MG1363 (van AlenBoerrigter et al, 1991; Tan et al, 1992) et Wg2 (Stroman, 1992). Dans les 2 cas, le gène a été isolé à partir d'une banque d'ADN de la souche de lactocoque chez Escherichia coli; dans le cas de MG1363, cette banque a été construite dans le phage $\lambda E M B L 3$ et a été criblée à l'aide d'anticorps spécifiques de l'enzyme, alors que dans le cas de $\mathrm{Wg} 2$, une sonde d'oligonucléotides dérivée de la séquence $\mathrm{N}$ terminale de l'enzyme a été utilisée pour le criblage de la banque. Le gène pepN correspond à une phase ouverte de lecture codant pour une protéine de 846 résidus dont la taille est en accord avec la masse moléculaire de $95 \mathrm{kDa}$ déterminée pour l'enzyme purifiée. La séquence en acides aminés de PepN déduite de la séquence nucléotidique présente des homologies importantes avec les métallo-aminopeptidases de mammifères (homme, rat, souris) avec plus de $25 \%$ d'identité. La région la plus conservée entre les différentes aminopeptidases est également homologue avec la famille des protéases neutres dépendantes du zinc telles que la thermolysine. Ce segment fait partie du site actif et contient le site de liaison du zinc. Aucune séquence signal n'a été identifiée à l'extrémité $\mathrm{N}$-terminale.

Le gène pepC codant pour la deuxième aminopeptidase de spécificité générale présente chez les lactocoques a été également cloné et séquencé récemment (Chapot-Chartier et al, 1993). Ce clonage a été réalisé par complémentation d'un mutant pepN de Ecoli déficient en activité aminopeptidase. Une banque d'ADN total de la souche de $L$ lactis subsp cremoris AM2 a été construite dans un vecteur plasmidique chez le mutant de $E$ coli et cette banque a été criblée par un test enzymatique sur boîte de Pétri à l'aide du substrat Leußnaphtylamide. Les anticorps dirigés contre l'enzyme PepC purifiée ont permis de montrer que l'un des clones sélectionnés exprimait PepC. Le fragment cloné contient une phase ouverte de lecture codant pour une protéine de 436 résidus dont la masse moléculaire correspond à celle d'une sous-unité de l'enzyme (50 $\mathrm{kDa})$. Aucune séquence signal n'est présente, ce qui indique une localisation intracellulaire de l'enzyme en accord avec les expériences de fractionnement cellulaire et les expériences d'immunocytochimie (Tan et al, 1992). La séquence en acides aminés déduite de la séquence nucléotidique, présente une forte homologie avec le site actif des protéases à cystéine avec conservation des résidus impliqués dans la catalyse (fig 1). En dehors du site actif, aucune homologie n'est observée avec la 
Tableau I. Peptidases isolées des lactocoques.

\begin{tabular}{|c|c|c|c|c|c|c|c|c|c|}
\hline Peptidases & Souches & Spécificité & $P M$ & $\begin{array}{l}\text { Nb de } \\
\text { sous-unités }\end{array}$ & Type & pHopt & $\begin{array}{l}\text { Topt } \\
\left({ }^{\circ} \mathrm{C}\right)\end{array}$ & $\begin{array}{l}\text { Gène } \\
\text { séquencé }\end{array}$ & Référence \\
\hline \multicolumn{10}{|c|}{ Aminopeptidases } \\
\hline Pep C & $\begin{array}{l}L \text { lactic subsp } \\
\text { cremoris AM2 }\end{array}$ & Large & 300 & 6 & Cystéine & 7 & 40 & Oui & Neviani et al (1989) \\
\hline \multirow[t]{3}{*}{ Pep N } & $\begin{array}{l}\text { L lactis subsp } \\
\text { cremoris Wg2 }\end{array}$ & Large & 95 & 1 & Metallo & 7 & 40 & Oui & Tan et Konings (1990) \\
\hline & $\begin{array}{l}\text { L lactis subsp } \\
\text { diacetylactis CNRZ } 267\end{array}$ & $7^{\text {Large }}$ & 85 & 1 & Metallo & 6,5 & 35 & Non & $\begin{array}{l}\text { Desmazeaud et Zevaco } \\
\text { (1979) }\end{array}$ \\
\hline & $\begin{array}{l}L \text { lactis subsp } \\
\text { cremoris AC1 }\end{array}$ & Large & 36 & 1 & Metallo & 7 & 40 & Non & Geis et al (1985) \\
\hline GAP I & $\begin{array}{l}\text { L lactis subsp } \\
\text { cremoris HP }\end{array}$ & $\begin{array}{l}\text { Asp et Glu } \\
\text { spécifique }\end{array}$ & 130 & 3 & Metallo & & $\begin{array}{l}50 \\
55\end{array}$ & Non & $\begin{array}{l}\text { Exterkate et } \\
\text { de Veer (1987a) }\end{array}$ \\
\hline GAP ॥ & $\begin{array}{l}\text { L lactis subsp } \\
\text { lactis NCDO } 712\end{array}$ & $\begin{array}{l}\text { Asp et Glu } \\
\text { spécifique }\end{array}$ & 245 & 6 & Metallo & 8 & 65 & Non & Niven (1991) \\
\hline \multicolumn{10}{|c|}{ Di- et tripeptidases } \\
\hline DIPI & $\begin{array}{l}\text { L lactis subsp } \\
\text { diacetylactis CNRZ } 267\end{array}$ & $\begin{array}{r}\text { spécifique } \\
7 \text { des Met-X }\end{array}$ & 51 & 1 & Metallo & $\begin{array}{l}7,5 \\
8\end{array}$ & & Non & $\begin{array}{l}\text { Desmazeaud et Zevaco } \\
\text { (1977) }\end{array}$ \\
\hline DIP ॥ & $\begin{array}{l}\text { L lactis subsp } \\
\text { cremoris } \mathrm{H} 61\end{array}$ & Large & 100 & & Metallo & 8 & & Non & Hwang et al (1981) \\
\hline DIP III & $\begin{array}{l}\text { L lactis subsp } \\
\text { cremoris Wg2 }\end{array}$ & Large & 49 & 1 & Metallo & 8 & 50 & Non & Van Boven et al (1988) \\
\hline TRP I & $\begin{array}{l}\text { L lactis subsp } \\
\text { diacetylactis CNRZ } 267\end{array}$ & $7^{\text {Large }}$ & 75 & & Metallo & 7 & 35 & Non & $\begin{array}{l}\text { Desmazeaud et Zevaco } \\
\text { (1977) }\end{array}$ \\
\hline TRP II & $\begin{array}{l}L \text { lactis subsp } \\
\text { cremoris Wg2 }\end{array}$ & Large & 105 & 2 & Metallo & 7,5 & 55 & Non & Bosman et al (1990) \\
\hline
\end{tabular}


Peptidases spécifiques des prolines

$X$-Prolyl dipeptidyl aminopeptidase

\begin{tabular}{|c|c|c|c|c|c|c|c|c|c|}
\hline Pep X & $\begin{array}{l}\text { L lactis subsp } \\
\text { cremoris } \mathrm{P} 8-2-47\end{array}$ & $\begin{array}{c}\text { Spécifique de } \\
\text { X-Pro en N termina }\end{array}$ & 180 & 2 & Sérine & 7 & $\begin{array}{l}45 \\
50\end{array}$ & Oui & $\begin{array}{l}\text { Kiefer-Partsch } \\
\text { et al (1989) }\end{array}$ \\
\hline XAPI & $\begin{array}{l}\text { L lactis subsp } \\
\text { cremoris AM2 }\end{array}$ & " & 117 & & Sérine & & & Non & Booth et al (1990b) \\
\hline Pep X & $\begin{array}{l}\text { L lactis subsp } \\
\text { lactis NCDO } 763\end{array}$ & " & 190 & 2 & Sérine & 8,5 & 40 & Oui & Zevaco et al (1990) \\
\hline XAP II & $\begin{array}{l}\text { L lactis subsp } \\
\text { lactis } \mathrm{H} 1\end{array}$ & $"$ & 150 & 2 & Sérine & & & Non & Lloyd et Pritchard (1991) \\
\hline \multicolumn{10}{|c|}{ Prolidase } \\
\hline PRD I & $\begin{array}{l}\text { L lactis subsp } \\
\text { cremoris } \mathrm{H} 61\end{array}$ & $\begin{array}{l}\text { Spécifique } \\
\text { de X-Pro }\end{array}$ & 43 & & Metallo & $\begin{array}{l}6,5 \\
7,5\end{array}$ & 40 & Non & Kaminogawa et al (1984) \\
\hline PRD II & $\begin{array}{l}\text { L lactis subsp } \\
\text { cremoris AM2 }\end{array}$ & $"$ & 42 & & Metallo & $\begin{array}{l}7,3 \\
9\end{array}$ & & Non & Booth et al (1990b) \\
\hline \multicolumn{10}{|c|}{ Proline iminopeptidase } \\
\hline PIP & $\begin{array}{l}\text { L lactis subsp } \\
\text { cremoris HP }\end{array}$ & $\begin{array}{l}\text { Spécifique de } \\
\text { Pro-X et Pro-X-Y }\end{array}$ & $y^{110}$ & 2 & Metallo & 8,5 & 37 & Non & $\begin{array}{l}\text { Baankreis et Exterkate } \\
\text { (1991) }\end{array}$ \\
\hline \multicolumn{10}{|c|}{ Endopeptidases } \\
\hline LEP I & $\begin{array}{l}L \text { lactis subsp } \\
\text { cremoris } \mathrm{H} 61\end{array}$ & Large & 98 & 1 & Metallo & $\begin{array}{l}7 \\
7,5\end{array}$ & 40 & Non & Yan et al (1987a) \\
\hline LEP \| & " & Large & 80 & 2 & Metallo & 6 & 37 & Non & Yan et al (1987b) \\
\hline LEP III & $\begin{array}{l}\text { L lactis subsp } \\
\text { cremoris Wg2 }\end{array}$ & Large & 70 & 1 & Metallo & $\begin{array}{l}6 \\
6,5\end{array}$ & $\begin{array}{l}30 \\
38\end{array}$ & Non & Tan et al (1991) \\
\hline
\end{tabular}




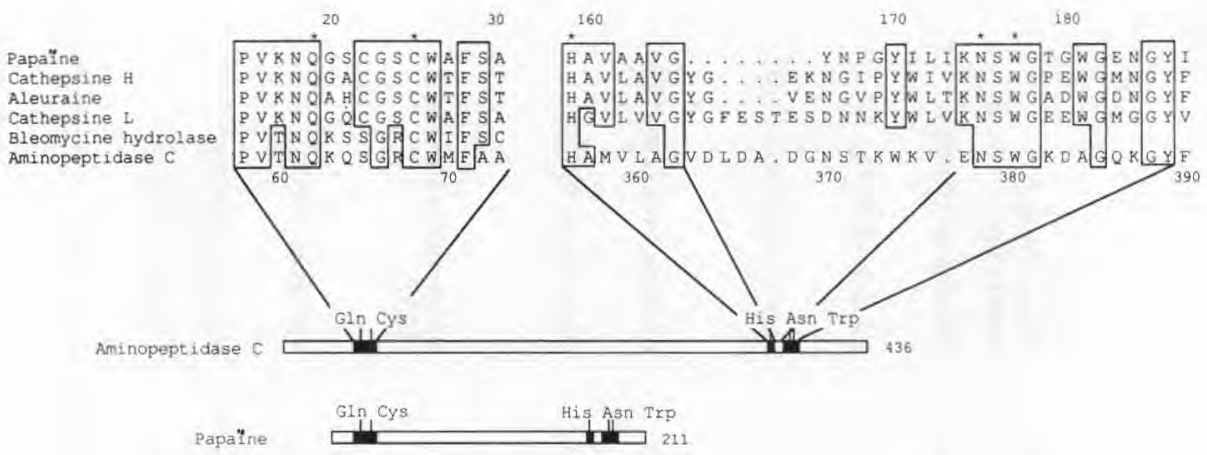

Fig 1. Conservation de la séquence en acides aminés entre l'aminopeptidase $\mathrm{PepC}$ de $L$ lactis subsp cremoris AM2 et les protéases à cystéine. En bas : régions conservées entre PepC et la papaïne. Les numéros à droite correspondent à la longueur de chaque polypeptide. Les parties en noir représentent les régions conservées dans les 2 séquences, et qui contiennent les résidus du site actif de la papaïne. En haut : alignement des régions conservées entre PepC et différentes protéases à cystéines. Les numéros sont relatifs à la position des acides aminés dans la papaïne (en haut) ou dans PepC (en bas). Les résidus conservés sont entourés. Les résidus du site actif de la papaïne sont indiqués par un astérisque.

papaine ni avec les autres protéases à cystéine sauf avec une enzyme de mammifère, la bléomycine hydrolase qui présente $34 \%$ d'identité avec PepC.

Les gènes pep $\mathrm{N}$ (van Alen-Boerrigter et al, 1991) ou pepC (non publié) ont été clonés dans le plasmide plL253 (Simon et Chopin, 1988) qui est un vecteur à haut nombre de copies des lactocoques. L'activité aminopeptidasique peut ainsi être surexprimée d'un facteur 20 .

\section{DI- ET TRIPEPTIDASES}

Deux dipeptidases capables d'hydrolyser une grande variété de dipeptides (exceptés ceux de type Gly-X ou Pro- $X$ ) et hydrolysant très mal les tripeptides ont été purifiées. Ce sont des métalloenzymes inhibées par les agents réducteurs des groupements thiol. L'une (DIP II) a été dé- crite par Hwang et al $(1981,1982)$ à partir de $L$ lactis subsp cremoris $\mathrm{H} 61$ comme étant un dimère de $100 \mathrm{kDa}$; l'autre (DIP III) par van Boven et al (1988) à partir de $L$ lactis subsp cremoris Wg2 comme un monomère de $49 \mathrm{kDa}$. Des différences ayant été notées dans leur spécificité et cinétique d'hydrolyse, il n'est pas possible d'affirmer qu'il s'agit de la même enzyme. Une autre dipeptidase, n'hydrolysant pas les tripeptides et ayant une préférence pour les dipeptides de type Met-X a aussi été purifiée à partir de $L$ lactis subsp diacetylactis CNRZ 267 et caractérisée. C'est une métalloenzyme d'un poids moléculaire de $51 \mathrm{kDa}$ (Desmazeaud et Zevaco, 1977).

Deux tripeptidases de spécificité large mais n'hydrolysant ni les dipeptides, ni les peptides de 4 acides aminés et plus ont été purifiées et caractérisées. Ce sont des métalloenzymes ; I'une (TRP I) d'un poids moléculaire de $75 \mathrm{kDa}$ est isolée de $L$ lac- 
tis subsp diacetylactis CNRZ 267 (Desmazeaud et Zevaco, 1979), I'autre (TRP II) est un dimère d'un poids moléculaire de $105 \mathrm{kDa}$, isolé de $L$ lactis subsp cremoris Wg2, qui n'est pas capable d'hydrolyser les tripeptides ayant une proline en position centrale (Bosman et al, 1990).

\section{PEPTIDASES SPECIFIQUES DES PROLINES}

Les caséines, source principale d'azote aminé pour les lactocoques, sont des protéines riches en proline, d'où la nécessité pour ces bactéries de posséder des peptidases spécifiques qui leur permettent de libérer cet acide aminé particulier. Plusieurs enzymes de ce type ont en effet été isolées de lactocoques.

\section{$X$-prolyl dipeptidyl aminopeptidase (X-Pro-Y-)}

\section{Caractérisation biochimique de l'enzyme}

Cette peptidase (PepX) libère des dipeptides X-Pro (et X-Ala mais avec une efficacité catalytique beaucoup plus faible, Lloyd et Pritchard, 1991) à partir de l'extrémité $\mathrm{N}$-terminale de chaînes peptidiques. Elle a été mise en évidence pour de nombreuses souches (Casey et Meyer, 1985; KieferPartsch et al, 1989; Zevaco et al, 1990; Booth et al, 1990b; Lloyd et Pritchard, 1991). Tous les auteurs s'accordent pour dire que c'est une enzyme à sérine de structure dimérique, chaque sous unité ayant un poids moléculaire d'environ 85 $\mathrm{kDa}$. L'intérêt de cette peptidase réside dans le fait qu'elle est tout à fait adaptée à I'hydrolyse des peptides de type X-Pro-YPro-Z-Pro.... provenant de la caséine $\beta$ et libérés par la protéinase de paroi.

\section{Clonage et séquençage du gène}

Le gène pepX codant pour l'enzyme $\mathrm{X}$ prolyl-dipeptidyl-aminopeptidase, a été le premier gène codant pour une peptidase qui a été isolé, le clonage ayant été réalisé simultanément par Nardi et al (1991) et Mayo et al (1991), à partir de 2 sousespèces de $L$ lactis.

Le gène de la souche $L$ lactis subsp lactis NCDO 763 a été cloné par complémentation d'un mutant négatif obtenu par mutagénèse à la nitrosoguanidine de la même souche, en utilisant un test d'activité sur colonies (Nardi et al, 1991). Ce gène a pu être exprimé chez Bacillus subtilis, qui ne possède pas cette activité. Le gène homologue de la souche de $L$ lactis subsp cremoris P8-2-47 a également été isolé, dans ce cas en criblant une banque d'ADN chez $E$ coli (Mayo et al, 1991). Les deux gènes ont été séquencés; ils contiennent 763 codons et sont identiques à $99 \%$. La taille de la protéine déduite de la séquence en acides aminés correspond à celle de l'enzyme purifiée ( $87 \mathrm{kDa})$. La séquence $\mathrm{N}$ terminale de la protéine purifiée est identique à celle de la séquence nucléotidique déduite du gène, ce qui indique que l'extrémité N-terminale de l'enzyme n'est soumise à aucune maturation. D'après la nature des inhibiteurs de son activité (DFP, PMSF...), l'enzyme apparaît comme une protéase à sérine. Cependant, PepX ne présente aucune homologie de séquence avec les 2 familles de protéases à sérine connues, dont les représentants les mieux étudiés sont la chymotrypsine pour les enzymes d'origine eucaryote et la subtilisine pour celles d'origine procaryote. Ces observations suggèrent que cette enzyme appartient à une nouvelle classe de protéases à sérine.

Comme dans le cas des 2 aminopeptidases PepN et PepC, PepX ne contient pas de séquence correspondant à une séquence signal ou une séquence d'ancrage 
membranaire. De telles séquences ont en revanche été précédemment identifiées chez les lactocoques dans le cas de la protéinase localisée dans la paroi, PrtP, et la protéine de maturation de cette protéase, PrtM, qui est une lipoprotéine membranaire.

En clonant le gène pepX dans le vecteur pGKV259 (van der Vossen et al, 1987), sous la dépendance d'un promoteur fort, l'activité X-prolyl-dipeptidylaminopeptidase a été surexprimée d'un facteur 10 (Nardi, communication personnelle).

\section{Prolidase (X-Pro)}

Complémentaire de la X-prolyl dipeptidyl aminopeptidase, une prolidase (PRD) capable d'hydrolyser les dipeptides X-Pro, a été mise en évidence, purifiée et caractérisée chez L lactis subsp cremoris $\mathrm{H} 61$ (Kaminogawa et al, 1984) et chez $L$ lactis subsp cremoris AM2 (Booth et al, 1990a). C'est une métalloenzyme de $42 \mathrm{kDa}$ qui n'hydrolyse ni les tripeptides, ni les dipeptides Gly-Pro.

\section{Proline iminopeptidase \\ (Pro-X ou Pro-X-Y) $\uparrow$ $\uparrow$}

Une proline iminopeptidase active sur des di- et tripeptides mais inactive sur des peptides plus longs ainsi que sur Pro-pnitroanilide a récemment été purifiée de $L$ lactis subsp cremoris HP (Baankreis et Exterkate, 1991). C'est une métalloenzyme de $110 \mathrm{kDa}$ de structure dimérique. Sa mise en évidence suggère qu'il existe différentes voies de libération de la proline. Booth et al (1990a) ont eux aussi, sur une autre souche, mis en évidence l'existence d'une faible activité prolinase sur le substrat Pro-Leu.

\section{ENDOPEPTIDASES}

Trois endopeptidases n'hydrolysant pas les protéines et leur préférant des chaînes peptidiques plus courtes ont été décrites chez les lactocoques mésophiles. Ce sont toutes des métalloenzymes et il est possible de les distinguer selon la taille des substrats qu'elles sont capables d'hydrolyser. LEP I (Yan et al, 1987b) n'hydrolyse ni les substrats trop courts ( 5 acides aminés) ni les substrats trop longs (25 acides aminés). LEP II (Yan et al, 1987a) et LEP III (Tan et al, 1991) sont moins strictes et peuvent hydrolyser à la fois des peptides de 5 acides aminés et des fragments peptidiques allant jusqu'à $3,5 \mathrm{kDa}$. Des séquences $\mathrm{N}$-terminales différentes pour ces 2 dernières enzymes suggèrent toutefois qu'elles sont différentes. LEP | et LEP || ont été isolées de $L$ lactis subsp cremoris H61 alors que LEP III l'a été de $L$ lactis subsp cremoris Wg2. II serait logique, bien qu'aucune preuve n'en ait encore été apportée qu'une ou plusieurs de ces endopeptidases intervienne dans la dégradation des peptides produits par la protéinase de paroi.

\section{Localisation et rôle dans la nutrition azotée}

Les peptides libérés après hydrolyse des caséines par la protéinase associée aux enveloppes sont, en grande majorité, d'une taille supérieure à 5 résidus (Monnet et al, 1986, 1989, 1992; Visser, 1988, 1991; Reid et al, 1991a et b). Ils doivent donc être hydrolysés préalablement à leur transport à travers la membrane, ce qui implique l'intervention de peptidases exocellulaires. La sécrétion de peptidases dans le milieu de culture n'a pu être mise en évidence chez les lactocoques ; les peptidases extracellulaires doivent donc être 
associées aux enveloppes bactériennes. Smid et al (1991) ont proposé un modèle dans lequel au moins 3 peptidases exocellulaires seraient nécessaires pour hydrolyser les peptides produits par la protéinase associée aux enveloppes. La caséine $\beta$, sous sa forme non micellaire, serait le substrat préférentiel de cette protéase et donc la principale source de peptides pour les lactocoques (Exterkate et de Veer, 1987b). Une aminopeptidase de spécificité large, PepN, libérerait des acides aminés libres (Ser, Thr, Val, Leu, Gin, Ala, Arg, Lys) ainsi que des dipeptides C-terminaux. Cette dernière est cependant incapable de libérer les acides aminés acides et ne coupe pas les liaisons de type X-Pro, aussi son action serait complétée par celle de PepA (qui libère Asp et Glu) et de PepX (qui libère des dipeptides de séquence $X$ Pro). L'action concertée de ces 3 enzymes serait donc suffisante pour dégrader les peptides produits par la protéinase associée aux enveloppes en acides aminés libres, di-tripeptides et très courts peptides capables d'utiliser les systèmes de transport présents dans la membrane (voir les revues de Konings et al, 1989 et 1991, pour la description de ces systèmes de transport). Dans ce schéma, la proline serait transportée essentiellement sous forme de dipeptides X-Pro et ensuite dégradée par la prolidase intracellulaire.

La localisation de plusieurs peptidases a été effectuée par fractionnement cellulaire. Les résultats ne sont toutefois pas toujours convergents et il convient d'être prudent dans leur interprétation car les fractions obtenues se contaminent mutuellement. L'utilisation d'une enzyme intracellulaire marqueur pour suivre la contamination de l'extrait de paroi par le cytoplasme n'est pas toujours un bon critère, cette enzyme pouvant être instable dans les conditions de l'extraction. A titre d'exemple, PepX a été décrite comme exocellulaire par Kiefer-Partsch et al (1989) alors que
Zevaco et al (1990) ont observé que $97 \%$ de l'activité se trouvait dans l'extrait cytoplasmique et seulement $3 \%$ dans l'extrait de paroi. La plupart des peptidases décrites ont toutefois été purifiées à partir d'extraits cytoplasmiques (PepX, PepC), de lysats ou de broyats de cellules ce qui suggère une localisation intracellulaire de ces enzymes.

Tan et al (1992) ont utilisé la microscopie électronique après marquage à l'or colloïdal pour localiser PepN, PepC, PepX, LEPIII et une tripeptidase. Toutes ces enzymes sont présentes dans le cytoplasme, cependant pour PepX, LEPIII et la tripeptidase un marquage périphérique est également observé. Ce marquage ne permet toutefois pas de dire si ces enzymes sont du côté externe ou du côté interne de la membrane.

Enfin, pour les peptidases dont le gène a été séquencé (PepX, PepN, PepC), il est intéressant de noter qu'aucune séquence signal n'a été observée, ce qui suggère fortement que ces enzymes sont intracellulaires. Leur présence à l'extérieur de la cellule impliquerait en effet l'existence d'un système de translocation à travers la membrane ne faisant pas intervenir de peptide signal. Un tel système a déjà été décrit chez certaines bactéries Gram négatives en particulier dans le cas de la translocation de l'hémolysine (Felmlee et al, 1985) mais n'a pas été démontré chez les bactéries Gram positives.

L'ensemble des données sur la localisation n'est donc pas entièrement compatible avec le modèle de Smid et al (1991) décrit précédemment, puisqu'au moins PepN et PepX semblent être intracellulaires. Plusieurs hypothèses alternatives peuvent être avancées. L'une est que les peptidases exocellulaires n'ont pas encore été mises en évidence, parce qu'elles sont particulièrement instables et ne résistent pas aux conditions d'extraction employées, 
ou parce qu'elles ne sont pas actives sur les substrats synthétiques généralement utilisés. Une autre hypothèse remet en cause l'existence de peptidases exocellulaires, la bactérie utiliserait alors uniquement les courts peptides (inférieurs à 5 résidus) produits par la protéinase associée aux enveloppes. Ces courts peptides sont toutefois peu abondants et il est peu probable qu'ils puissent apporter les acides aminés indispensables en quantité suffisante. Dans une dernière hypothèse, la lyse d'une faible proportion des cellules serait à l'origine de la liberation d'une activité exopeptidasique dans le milieu de culture. Cette activité hydrolyserait les peptides produits par la protéinase associée aux enveloppes. II faut cependant noter que les dosages d'activités peptidasiques dans le milieu de culture au cours de la croissance n'ont pas permis de révéler cette libération.

Comme on le voit, aucune des hypothèses présentées n'est vraiment démontrée et des études sont encore nécessaires pour obtenir une vision claire du mécanisme de la nutrition azotée des lactocoques.

\section{REFERENCES}

Baankreis R, Exterkate FA (1991) Characterisation of a peptidase from Lactococcus lactis subsp cremoris HP that hydrolyses dipeptides and tripeptides containing proline or hydrophobic residues as the aminoterminal amino acid. Syst Appl Microbiol 14, 317-323

Booth M, Donnelly WJ, Fhaolain IN, Jennings PV, O'Cuinn G (1990a) Proline-specific peptidases of Streptococcus cremoris AM2. I Dairy Res 57, 79-88

Booth M, Fhaolain IN, Jennings PV, O'Cuinn G (1990b) Purification and characterization of a post-proline dipeptidyl aminopeptidase from Streptococcus cremoris AM2. I Dairy Res 57, 89-99

Bosman BW, Tan PST, Konings WN (1990) Purification and characterization of a tripepti- dase from Lactococcus lactis subsp cremoris Wg2. Appl Environ Microbiol 56, 1839-1843

Casey MG, Meyer J (1985) Presence of Xprolyl-dipeptidyl-peptidase in lactic acid bacteria. J Dairy Sci 68, 3212-3215

Chapot-Chartier MP, Nardi M, Chopin MC, Chopin A, Gripon JC (1993) Cloning and sequencing of pepC, a cysteine aminopeptidase gene from Lactococcus lactis subsp cremoris AM2. Appl Environ Microbiol 59, 330-333

Cliffe AJ, Law BA (1979) An electrophoretic study of peptidases in starter streptococci and in Cheddar cheese, J Appl Bacteriol 47, 65-73

Desmazeaud MJ, Zevaco C (1977) General properties and substrate specificity of an intracellular soluble dipeptidase from Streptococcus diacetylactis. Ann Biol Anim Biochim Biophys 17, 723-736

Desmazeaud MJ, Zevaco C (1979) Isolation and general properties of two intracellular aminopeptidases of Streptococcus diacetylactis. Milchwissenschaft 34,606-610

Exterkate FA, de Veer GJCM (1987a) Purification and some properties of a membranebound aminopeptidase A from Streptococcus cremoris. Appl Environ Microbiol 53, 577-583

Exterkate FA, de Veer GJCM (1987b) Optimal growth of Streptococcus cremoris HP in milk is related to $\beta$ - and $\mathrm{k}$ - degradation. Appl Microbiol Biotechnol 25, 471-475

Felmlee T, Pellett S, Lee EY, Welch RA (1985) Escherichia coli hemolysin is released extracellularly without cleavage of a signal peptide. J Bacteriol 163, 88-93

Geis A, Bockelmann W, Teuber M (1985) Simultaneous extraction and purification of a cell wall-associated peptidase and $\beta$-casein specific protease from Streptococcus cremoris AC1. Appl Microbiol Biotechnol 23, 79-84

Hwang I, Kaminogawa S, Yamauchi K (1981) Purification and properties of a dipeptidase from Streptococcus cremoris. Agric Biol Chem 45, 159-165

Hwang I, Kaminogawa S, Yamauchi K (1982) Kinetic properties of a dipeptidase from Streptococcus cremoris. Agric Biol Chem 46, 3049-3053

Kaminogawa S, Ninomiya T, Yamauchi K (1984) Aminopeptidase profiles of lactic streptococci. J Dairy Sci 67, 2483-2492 
Kiefer-Partsch B, Bockelmann W, Geis A, Teuber M (1989) Purification of an X-prolyldipeptidyl aminopeptidase from the cell wall proteolytic system of Lactococcus lactis subsp cremoris. Appl Microbiol Biotechnol 31, 75-78

Kok J, Venema G (1988) Genetics of proteinases of lactic acid bacteria. Biochimie 70,475-488

Konings WN, Poolman, B, Driessen AJM (1989) Bioenergetics and solute transport in lactococci. CRC Crit Rev Microbiol 16, 419-476

Konings WN, Smid EJ, Laan H, Driessen AJM (1991) From casein to cheese : the role of Lactococcus lactis. Food Biotechnol 5, $263-$ 277

Law BA (1979) Extracellular peptidases in group $\mathrm{N}$ streptococci used as cheese starters. J Appl Bacteriol 46, 455-463

Lloyd RJ, Pritchard GG (1991) Characterization of X-prolyl dipeptidyl aminopeptidase from Lactococcus lactis subsp lactis. J Gen Microbiol 137, 49-55

Mayo B, Kok J, Venema K, Bockelmann W, Teuber M, Renke H, Venema G (1991). Molecular cloning and sequence analysis of the $X$ prolyl dipeptidyl aminopeptidase gene from Lactococcus lactis subsp cremoris. Appl Environ Microbiol 57, 38-44

Monnet V, Le Bars D, Gripon JC (1986) Specificity of a cell wall proteinase from Streptococcus lactis NCDO 763 towards bovine $\beta$ casein. FEMS Microbiol Lett 36, 127-131

Monnet V, Bockelmann W, Gripon JC, Teuber M (1989) Comparison of cell wall proteinases from Lactococcus lactis subsp cremoris AC1 and Lactococcus lactis subsp lactis NCDO 763. II. Specificity towards bovine $\beta$-casein. Appl Microbiol Biotechnol 31, 112-118

Monnet V, Ley JP, Gonzalez S (1992) Substrate specificity of the cell envelope-located proteinase of Lactococcus lactis subsp lactis NCDO-763. Int J Biochem 24, 707-718

Mou L, Sullivan JJ, Jago GR (1975) Peptidase activities in group $\mathrm{N}$ streptococci. J Dairy Res 42, 147-155

Nardi M, Chopin MC, Chopin A, Cals MM, Gripon JC (1991) Cloning and DNA sequence analysis of an X-prolyl-dipeptidyl aminopeptidase from Lactococcus lactis subsp cremoris AM2. Appl Environ Microbiol 57, 2308-2314
Neviani E, Boquien CY, Monnet V, Phan Thanh L, Gripon JC (1989) Purification and characterization of an aminopeptidase from Lactococcus lactis subsp cremoris AM2. Appl Environ Microbiol 55, 2308-2314

Niven GW (1991) Purification and characterization of aminopeptidase A from Lactococcus lactis subsp lactis NCDO 712. J Gen Microbiol 137, 1207-1212

Reid JR, Moore $\mathrm{CH}$, Midwinter GG, Pritchard GG (1991a) Action of a cell wall proteinase from Lactococcus lactis subsp cremoris SK11 on bovine $\alpha$ 1-casein. Appl Microbiol Biotechnol 35, 222-227

Reid JR, $\mathrm{Ng} \mathrm{KH}$, Moore $\mathrm{CH}$, Coolbear T, Pritchard GG (1991b) Comparison of bovine $\beta$ casein hydrolysis by $\mathrm{PI}$ and PIII type proteinases from Lactococcus lactis subsp cremoris. Appl Microbiol Biotechnol 36,344-351

Schmidt RH, Morris HA, McKay LL (1977) Cellular location and characteristics of peptidase enzymes in lactic streptococci. $J$ Dairy Sci 60, 710-717

Simon, D, Chopin, A (1988) Construction of a vector plasmid family and its use for molecular cloning in Streptococcus lactis. Biochimie 70, 559-566

Smid EJ, Poolman B, Konings WN (1991) Casein utilization by lactococci. Appl Environ Microbiol 57, 2447-2452

Stroman P (1992) Sequence of a gene (lap) encoding a 95.3-kDa aminopeptidase from $\mathrm{Lac}$ tococcus lactis subsp cremoris Wg2. Gene $113,107-112$

Tan PST, Konings WN (1990) Purification and characterization of an aminopeptidase from Lactococcus lactis subsp cremoris Wg2. Appl Environ Microbiol 56, 526-532

Tan PST, Pos KM, Konings WN (1991) Purification and characterization of an endopeptidase from Lactococcus lactis subsp cremoris Wg2. Appl Environ Microbiol 57, 35933599

Tan PST, Chapot-Chartier MP, Pos KM, Rousseau M, Boquien CY, Gripon JC, Konings WN (1992) Localization of peptidases in lactococci. Appl Environ Microbiol 58, 285-290

Tan PST, van Alen-Boerrigter IJ, Poolman B, Siezen RJ, de Vos WM, Konings WN Characterization of the Lactococcus lactis pepN gene encoding an aminopeptidase homolo- 
gous to mammalian aminopeptidase N. Soumis pour publication

Thomas TD, Mills OE (1981) Proteolytic enzymes of starter bacteria. Neth Milk Dairy J $35,255-273$

Thomas TD, Pritchard GG (1987) Proteolytic enzymes of dairy starter cultures. FEMS Microbiol Rev 46, 245-268

van Alen-Boerrigter IJ, Baankreis R, de Vos WM (1991) Characterization and overexpression of the Lactococcus lactis pepN gene and localization of its product, aminopeptidase N. Appl Environ Microbiol 57, 25552561

van Boven A, Tan PST, Konings WN (1988) Purification and characterization of a dipeptidase from Streptococcus cremoris Wg2. Appl Environ Microbiol 54, 43-49

van der Vossen JMBM, van der Lelie D, Venema G (1987). Isolation and characterization of Lactococcus lactis subsp cremoris Wg2specific promoters. Appl Environ Microbiol $53,2452-2457$
Visser S, Exterkate FA, Slangen CJ, de Veer GJCM (1988) Action of a cell wall proteinase (PI) from Streptococcus cremoris HP on bovine B-casein. Appl Microbiol Biotechnol 29, 61-66

Visser S, Robbes AJPM , Slangen CJ (1991) Specificity of a cell-envelope-located proteinase (PIII type) from Lactococcus lactis subsp cremoris am1 in its action on bovine B-casein. Appl Microbiol Biotechno/ 35, 477-483

Yan TR, Azuma S, Kaminogawa S, Yamauchi K (1987a) Purification and characterization of a novel metalloendopeptidase from Streptococcus cremoris H61. Eur J Biochem 163, 259265

Yan TR, Azuma N, Kaminogawa S, Yamauchi K (1987b) Purification and characterization of a substrate-size-recognizing metalloendopeptidase from Streptococcus cremoris H61. Appl Environ Microbiol 53, 2296-2302

Zevaco C, Monnet V, Gripon JC (1990) Intracellular X-prolyl dipeptidyl peptidase from Lactococcus lactis subsp lactis - purification and properties. J Appl Bacteriol 68, 357-366 